\title{
Up-regulating autophagy by targeting the mTOR-4EBP1 pathway: a possible mechanism for improving cardiac function in mice with experimental dilated cardiomyopathy
}

\author{
Bo Jin ${ }^{1}$, Haiming Shi ${ }^{1}$, Jun Zhu ${ }^{1}$, Bangwei Wu ${ }^{1 *}$ (D) and Quzhen Geshang ${ }^{2}$
}

\begin{abstract}
Background: Autophagy plays a crucial role in the pathological process of cardiovascular diseases. However, little is known about the pathological mechanism underlying autophagy regulation in dilated cardiomyopathy (DCM).

Methods: We explored whether up-regulating autophagy could improve cardiac function in mice with experimental DCM through the mTOR-4EBP1 pathway. Animal model of DCM was established in BALB/C mice by immunization with porcine cardiac myosin. Both up- or down-regulation of autophagy were studied by administration of rapamycin or 3-MA in parallel. Morphology, Western blotting, and echocardiography were applied to confirm the pathological mechanisms.

Results: Autophagy was activated and autophagosomes were significantly increased in the rapamycin group. The collagen volume fraction (CVF) was decreased in the rapamycin group compared with the DCM group (9.21 \pm $0.82 \%$ vs $14.38 \pm 1.24 \%, P<0.01$ ). The expression of $p-m T O R$ and $p-4 E B P 1$ were significantly decreased in rapamycininduced autophagy activation, while the levels were increased by down-regulating autophagy with 3-MA. In the rapamycin group, the LVEF and FS were significantly increased compared with the DCM group $(54.12 \pm 6.48 \%$ vs $45.29 \pm 6.68 \%, P<0.01 ; 26.89 \pm 4.04 \%$ vs $22.17 \pm 2.82 \%, P<0.05)$. As the inhibitor of autophagy, 3-MA aggravated the progress of maladaptive cardiac remodeling and declined cardiac function in DCM mice.
\end{abstract}

Conclusions: The study indicated a possible mechanism for improving cardiac function in mice with experimental DCM by up-regulating autophagy via the mTOR-4EBP1 pathway, which could be a promising therapeutic strategy for DCM.

Keywords: Autophagy, mTOR, Cardiac function, Dilated cardiomyopathy

\section{Background}

Dilated cardiomyopathy (DCM) is one of the most common cardiomyopathy worldwide characterized by left ventricular dilation and decline in contraction function, which is the third leading cause of congestive heart failure [1-3]. Recent studies of pathological mechanisms underlying heart failure focus on structural changes in

\footnotetext{
* Correspondence: wbwhsh@aliyun.com

1 Department of Cardiology, Huashan Hospital, Fudan University, 12 Middle Urumqi Road, Shanghai 200040, China

Full list of author information is available at the end of the article
}

cardiomyocytes and extracellular matrix to explain the deleterious contractile function $[4,5]$. However, the exact molecular mechanism in the onset and progression of DCM is still unclear.

Cardiovascular diseases including hypertrophic and ischemic cardiomyopathies are increasingly being reported to accumulate misfolded proteins and damaged organelles. As the highly conserved pathway, autophagy plays a crucial role in the pathological process of cardiovascular diseases. Previous studies indicated that autophagy is activated in maladaptive

(c) The Author(s). 2020 Open Access This article is distributed under the terms of the Creative Commons Attribution 4.0 International License (http://creativecommons.org/licenses/by/4.0/), which permits unrestricted use, distribution, and 
cardiac remodeling of chronic heart failure [6-8]. However, little is known about the pathological mechanism underlying autophagy regulation by pharmacological interventions in dilated cardiomyopathy. There are still many unanswered questions and points of confusion that have yet to be resolved. Therefore, an indepth investigation into the molecular mechanism is vital to therapeutic interventions in the field. Here, we focuses on whether regulating autophagy could improve cardiac function in DCM mice through the mTOR-4EBP1 pathway.

To the best of our knowledge, little information is available to confirm whether up-regulating autophagy could improve cardiac function in DCM mice. In the present study, we propose that autophagy regulation plays a crucial role in determining cardiac function in mice with experimental DCM through the mTOR4EBP1 pathway. Therefore, we pinpointed that upregulating autophagy by targeting the mTOR-4EBP1 pathway as the possible molecular mechanisms in cardio-protection in DCM mice. Both up- or downregulation of autophagy were investigated by administration of rapamycin or 3-methyadenine (3-MA) in parallel. Based on these findings, modulating autophagy could be a potential therapeutic target to minimize myocardial injury and optimize the restoration of cardiac function [9-11].

\section{Methods}

\section{Animal models and experimental design}

All animal experiments were approved by the Animal Care and Utilization Committee of Fudan University (201802021S). We obtained the male BALB/c mice aged 6 weeks from Fudan University Experimental Animal Center. The animal model of DCM was established in BALB/c mice by injection with porcine cardiac myosin (Sigma). Cardiac myosin was emulsified with an equal volume of complete Freund's adjuvant (Sigma) to the concentration of 2 $\mathrm{mg} / \mathrm{ml}$. The cardiac myosin was subcutaneously injected into the groin of $\mathrm{BALB} / \mathrm{c}$ mice twice at days 0 and day 7. The total dose for DCM induction was $0.2 \mathrm{mg}$ per mouse. The mice in the control group were injected with complete Freund's adjuvant as the vehicle. As previously reported [12], we confirmed myosininduced DCM model by histomorphological study and echocardiographic assessments in the present study. Additional eight normal mice and twenty-four DCM mice were divided into the following four experimental groups as follows: control group (normal + PBS), DCM group (DCM + $\mathrm{PBS}$ ), rapamycin group (DCM + rapamycin) and 3-MA group (DCM + 3-MA). Eight weeks after immunization, rapamycin was then administered at a dose of $2 \mathrm{mg} / \mathrm{kg} / \mathrm{d}$ for 2 weeks. The mice in 3-MA group received 3-MA at a dose of $15 \mathrm{mg} / \mathrm{kg} / \mathrm{d}$, while the mice in the control group were injected with PBS alone. After intraperitoneal injection of sodium pentobarbital $(75 \mathrm{mg} / \mathrm{kg}$ body weight), all mice were sacrificed by cervical dislocation while anesthetized.

\section{Echocardiographic measurements}

M-mode transthoracic echocardiography was performed using a $30-\mathrm{MHz}$ imaging transducer to evaluate the cardiac function. The mice in the four experimental groups were anesthetized with $2 \%$ isoflurane and their chests were epilated. M-mode images were obtained at the level of papillary muscles in the long-axis view. The left ventricular ejection fraction (LVEF), fractional shortening (FS), left ventricular end-diastolic dimension (LVEDD), and left ventricular end-diastolic volume (LVEDV) were measured, which were acquired by the technician who was blinded to the present experimental groups.

\section{Histopathology image analysis}

Myocardial tissues were obtained and fixed in $4 \%$ formaldehyde, embedded in paraffin and cut into $5 \mu \mathrm{m}$ thick slices. Specimens were treated and stained with picrosirius red, and microscopic images were observed. The collagen volume fraction (CVF) was measured by quantitative morphometry of specimens with IMS Cell Image Analysis System (Shen Teng, Shanghai, China). Morphological changes were investigated under confocal scanning microscope (Leica, TCS-SP2, Germany). For quantitation of cardiac fibrosis areas, 5 random fields of view per mouse were evaluated for CVF analysis across the left ventricular section (Each group, $n=8$ ). Consequently, there were 40 quantitative data for statistical analysis in each group.

\section{Transmission electron microscopy evaluation}

Transmission electron microscopy (TEM) for morphological evaluation was performed at Electron Microscopy Core Laboratory, Shanghai medical college, Fudan University (Philips CM120, Nethelands), according to standard operating procedures. As previously reported for morphological TEM [13], cardiac tissues were fixed in $2.5 \%$ glutaraldehyde in phosphate buffer overnight at $4{ }^{\circ} \mathrm{C}$. After sample preparation, $90-100 \mathrm{~nm}$ thick sections were mounted onto a 200 mesh copper grid and examined under a Philips CM120 electron microscope. The pathological alterations of cardiomyocyte nucleus, mitochondria, myocardial fibers, and autophagosomes were evaluated in the four groups. 


\section{Western blotting assays}

After being harvested, the left ventricular myocardium specimens were stored at $-80{ }^{\circ} \mathrm{C}$. Proteins were extracted from the myocardial tissues homogenized in RIPA Lysis (Beyotime) and Extraction Buffer with a protease inhibitor cocktail, and proteins were quantified using the bicinchoninic acid method according to the manufacturer's instructions. The total of $25 \mu \mathrm{g}$ protein samples were loaded into 8\% SDSPAGE gels for electrophoresis then transferred to PVDF membranes over night at $30 \mathrm{~V}$. Antibodies specific for LC3 II (dilution 1:1000; Cell Signaling), p-mTOR (dilution 1:1000; Cell Signaling), and p4EBP1 (dilution 1:1000; Cell Signaling) were incubated at $4{ }^{\circ} \mathrm{C}$ overnight, and GAPDH (dilution 1: 5000; Santa Cruz) was used as a loading control to normalize gel loading and protein expression. HRPconjugated secondary antibodies plus ECL were incubated at $37^{\circ} \mathrm{C}$ for $1 \mathrm{~h}$ for protein visualization. The densitometric values of immunoreactive bands were measured using Image J (NIH, USA).

\section{Statistical analysis}

The data are presented as mean \pm standard deviation. Values of $P$ less than 0.05 were considered statistically significant. Normal distribution was confirmed in four experimental groups, and differences in means between two groups were analyzed by unpaired Student's t test when the data were normally distributed. Multiple group comparison was performed by one-way ANOVA followed by Newman-Keuls multiple comparison test. GraphPad Prism version 6.0 software (GraphPad Software Inc., USA) was used for data analysis.

\section{Results}

\section{General characteristics}

The animal model was successfully established in male BALB/c mice, and twenty-four DCM mice were randomly divided into DCM group, rapamycin group, and 3-MA group equally. Furthermore, eight normal mice in the control group were administered with Freund's adjuvant alone. No significant difference was found in the body weight, heart weight and heart weight/body weight $(\mathrm{HW} / \mathrm{BW})$, although a tendency was found that the body weight was slightly decreased in the 3-MA group, it did not reach the statistically significant level (Table 1).

\section{Modulating autophagy and morphological evaluation}

The experimental model of DCM was established in $\mathrm{BALB} / \mathrm{c}$ mice by immunization with porcine cardiac myosin. Histochemical analysis with picrosirius red staining indicated that there was a significant increase of CVF in the DCM group compared with the control
Table 1 The general characteristics of the four experimental groups

\begin{tabular}{lllll}
\hline & $\begin{array}{l}\text { Control } \\
\text { group }\end{array}$ & $\begin{array}{l}\text { DCM } \\
\text { group }\end{array}$ & $\begin{array}{l}\text { Rapamycin } \\
\text { group }\end{array}$ & $\begin{array}{l}\text { 3-MA } \\
\text { group }\end{array}$ \\
\hline Number of death & 0 & 1 & 0 & 0 \\
Body weight (g) & $19.87 \pm 2.40$ & $19.72 \pm 2.22$ & $19.69 \pm 2.16$ & $19.59 \pm 2.27$ \\
Heart weight (g) & $0.22 \pm 0.02$ & $0.21 \pm 0.01$ & $0.22 \pm 0.02$ & $0.23 \pm 0.03$ \\
HW/ BW (mg/g) & $11.09 \pm 1.25$ & $10.94 \pm 1.08$ & $11.15 \pm 1.21$ & $11.53 \pm 1.33$ \\
\hline HW/ BW Heart weight/ Body weight $(\mathrm{mg} / \mathrm{g})$ Each group,$n=8$ &
\end{tabular}

group, revealing cardiac fibrosis in DCM mice. Figure 1 indicated that the CVF was significantly decreased in the rapamycin group than the DCM group (9.21 \pm $0.82 \%$ vs $14.38 \pm 1.24 \%, P<0.01)$. However, the CVF was increased to $17.68 \pm 1.81 \%$ by down-regulating autophagy in the 3-MA group compared with the DCM group $(P<0.05)$.

For morphological TEM, normally arranged myofibrils within the sarcomeres with defined Z-bands were observed in the control group. Autophagy was significantly activated and autophagosomes could be confirmed in mice with experimental DCM, and sarcomeric disarray and myofibrillar lysis could be observed. As shown in Fig. 2, double membrane autophagosomes were significantly increased in the rapamycin group compared with the DCM group $(P<$ 0.001 ). We inhibited the autophagy activation by 3 MA and verified that the number of autophagosomes was statistically decreased compared with the DCM group, and the sarcomeric disarray failed to get reversed.

\section{Modulating autophagy and mTOR-4EBP1 pathway}

The conversion of LC3 I to LC3 II form is recognized as indicators of autophagy activation. To validate the relationship of autophagy and mTOR-4EBP1 pathway, the p-mTOR and the downstream molecule of p4EBP1 were measured. Autophagy and mTOR-4EBP1 pathway were regulated in mice with experimental DCM by administration of rapamycin or 3-MA in parallel. Our study indicated that rapamycin-induced inhibition of mTOR-4EBP1 pathway, shown as decreased p-mTOR and p-4EBP1 expression compared with the DCM group. The increased expression of LC3 II indicated the activation of autophagy in the rapamycin group. With the administration of 3-MA, protein levels of p-mTOR and p-4EBP1 were significantly increased, whereas the expression of LC3 II was decreased in the 3-MA group (Fig. 3).

\section{Modulating autophagy and cardiac function}

We next examined whether the cardiac function was improved in mice with experimental DCM by upregulating autophagy. Therefore, M-mode images were 


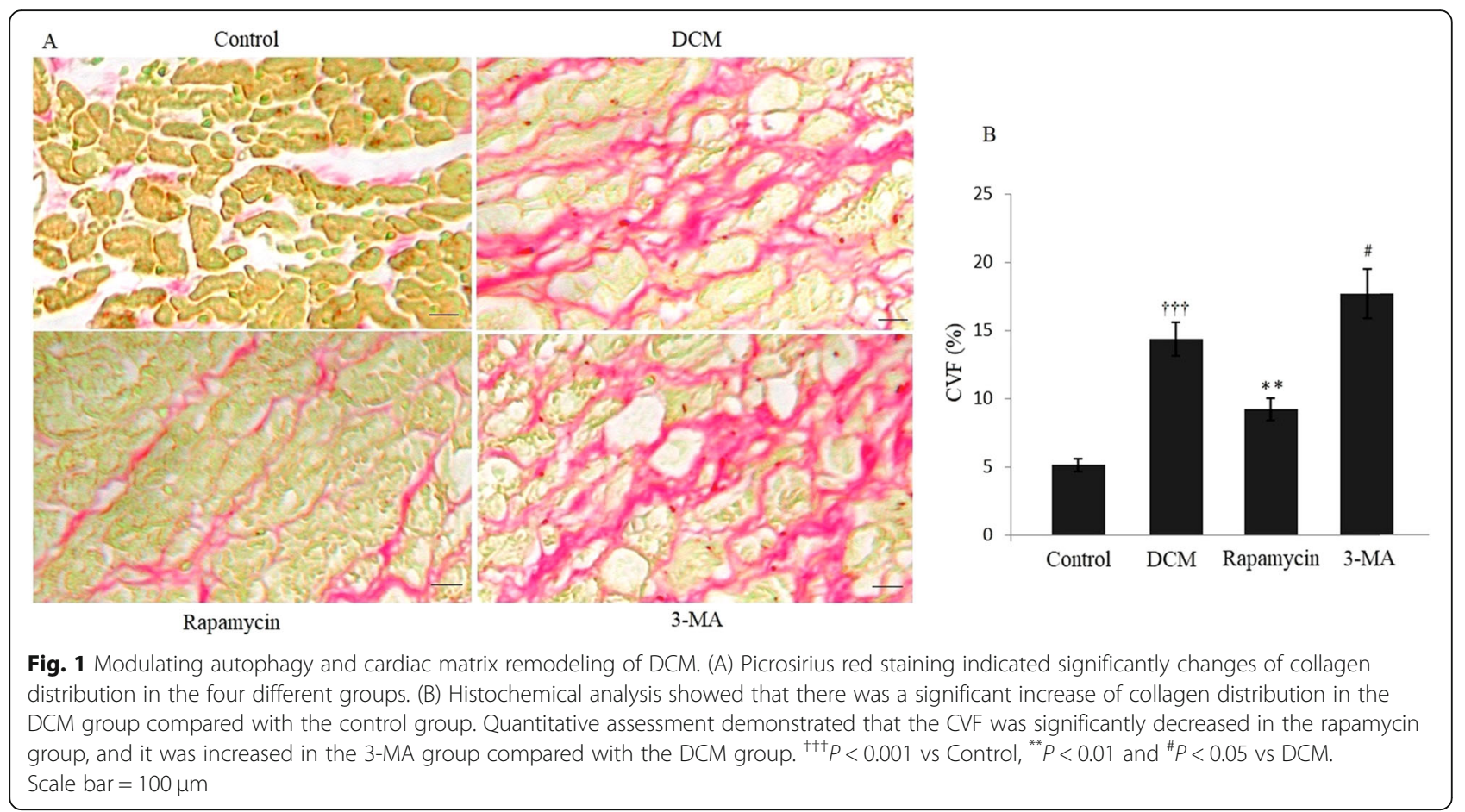

obtained at the level of papillary muscles in the longaxis view (Fig. 4). As summarized in Fig. 5, cardiac function differed significantly among the four groups. In the DCM group, the LVEF and FS significantly deteriorated compared with the control group. In the rapamycin group, the parameters were significantly increased compared with the DCM group (54.12 \pm
$6.48 \%$ vs $45.29 \pm 6.68 \%, \quad P<0.01 ; \quad 26.89 \pm 4.04 \% \quad$ vs $22.17 \pm 2.82 \%, P<0.05$ ), although cardiac function was still lower than the control group. Furthermore, the LVEDD and LVEDV significantly decreased following down-regulation of mTOR-4EBP1 pathway to activate autophagy. However, 3-MA-induced inhibition of autophagy provided a negative effect to promote the

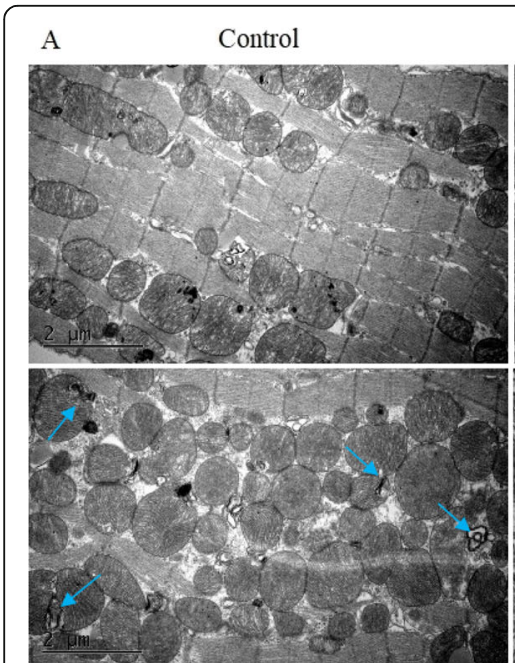

Rapamycin

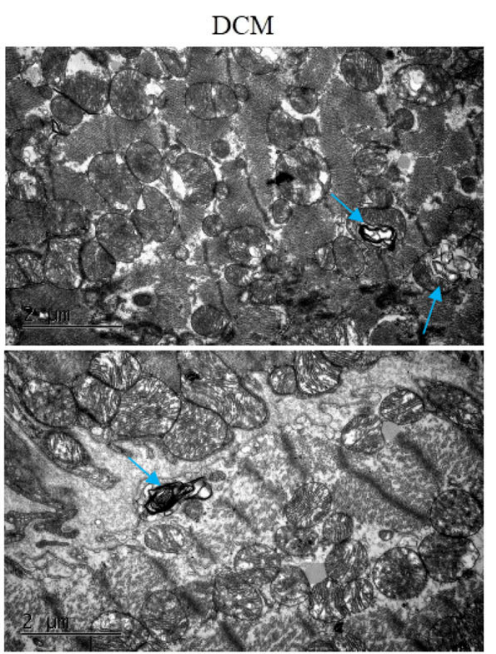

3-MA

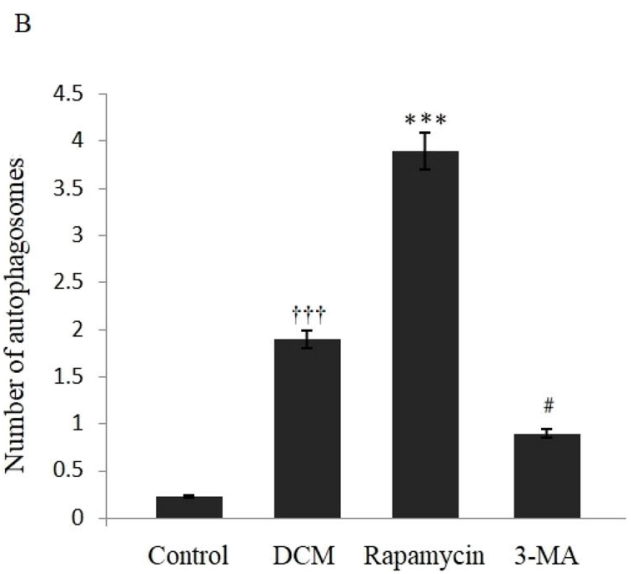

Control DCM Rapamycin 3-MA

Fig. 2 Transmission electron microscopy assessment for modulating autophagy. (A) Transmission electron microscopy indicated significant changes of autophagosomes in the four different groups. (B) Transmission electron microscopy showed that there was a significant increase of autophagosomes in the DCM group compared with the control group. Quantitative assessment demonstrated that autophagosomes were significantly increased in the rapamycin group, and they were decreased in the 3-MA group compared with the DCM group. ${ }^{++\dagger} P<0.001$ vs Control, ${ }^{* * *} P<0.01$ and ${ }^{\#} P<0.05$ vs DCM. The arrows indicated the double membrane autophagosomes in the different groups 

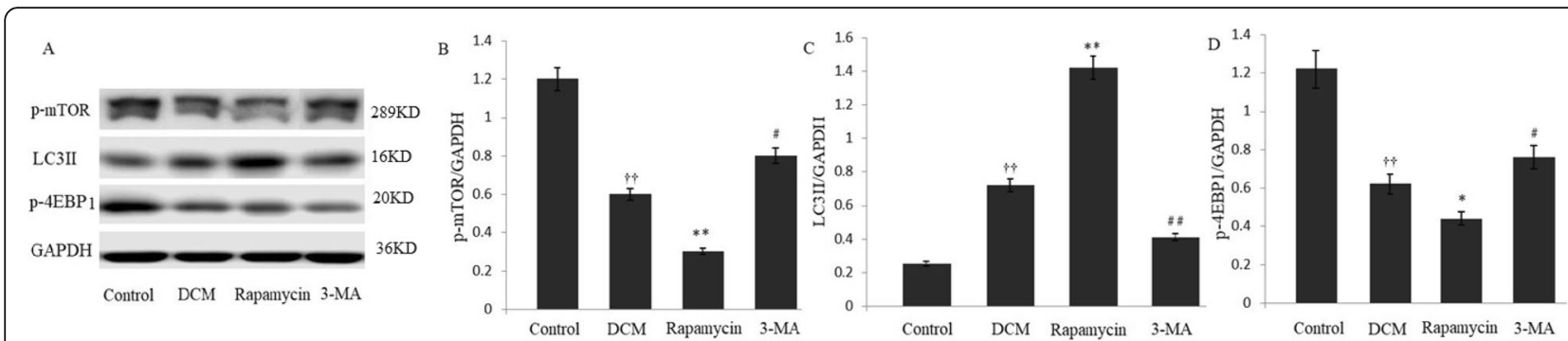

Fig. 3 Modulating autophagy and the mTOR-4EBP1 pathway. a-d The expression levels of p-mTOR and p-4EBP1 were significantly decreased in rapamycin-induced autophagy activation, and the effects were significantly increased by down-regulating autophagy with 3MA. The increased expression of LC3 II indicated the activation of autophagy in the rapamycin group, whereas the expression of LC3 II was decreased in the 3-MA group. ${ }^{\dagger \dagger} P<0.01$ vs Control, ${ }^{* *} P<0.01,{ }^{*} P<0.05,{ }^{\# \#} P<0.01$, and ${ }^{\#} P<0.05$ vs DCM. Each experiment was conducted 3 times in triplicate

maladaptive cardiac remodeling, possibly in part, by up-regulation of mTOR-4EBP1 pathway involved in the pathological process of DCM.

\section{Discussion}

Experimental autoimmune myocarditis in mice is a typical animal model which mimics the pathophysiological process of DCM. For the first time we reported the cardio-protective effects of rapamycin-induced autophagy activation, which contributed to improve cardiac function in DCM mice via regulating the mTOR-4EBP1 pathway. The biological effects were tested in experimental DCM mice by administration of rapamycin or 3MA respectively. We confirmed that autophagy was directly activated by down-regulating the mTOR-4EBP1 pathway, which increased the expression of LC3 II and the formation of autophagosomes. Our study indicated that up-regulating autophagy could be a promising therapeutic strategy to improve cardiac function for the pathological progression of DCM.

Autophagy is a highly conserved cellular recycling process, which not only plays an important role in cellular homeostasis but also participates in physiological processes [14, 15]. Autophagy degrades the recycling material in the cell while the former makes it through the formation of double-membrane vesicles that fuse with the lysosomal [16-18]. It plays the major role of catabolic mechanism degenerating and recycling longlived protein and organelles involving in physiological and pathological process. Accordingly, dysfunction of this process contributes to the pathological process of cardiovascular diseases.

The mTOR pathway is a well-known negative regulator of autophagic activity, which has been established to regulate cell growth, proliferation, and metabolism [19-21]. Our previous study indicated that autophagic activity was up-regulated in a rat model of early-stage dilated cardiomyopathy, which was a part of the reparative processes during DCM progression [22]. As a mTOR inhibitor, rapamycin can dephosphorylate the downstream effectors such as 4EBP1. Furthermore, $4 \mathrm{EBP} 1$ is a translation regulator, its dephosphorylation by mTOR inhibitors suppresses overall cellular protein synthesis and induces autophagy [23]. In our present study, rapamycin-induced autophagy activation successfully reversed myocardial fibrosis and improved cardiac function in DCM mice. In contrast, down-regulating autophagy inhibited the formation of autophagosomes in the 3-MA group, which induced severe myocardial fibrosis and decreased cardiac function.

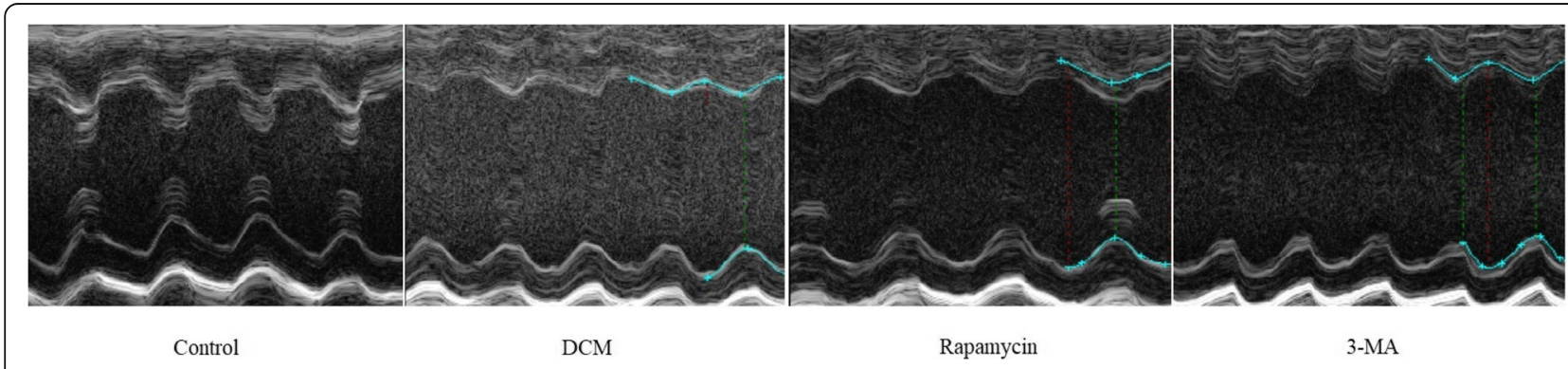

Fig. $4 \mathrm{M}$-mode ultrasound images at the level of papillary muscles in the long-axis view. The M-mode echocardiography evaluation included the following four experimental groups as follows: control group, DCM group, rapamycin group, and 3-MA group. The red lines and blue lines indicated the LVEDD and LVESD respectively. Each group, $n=8$ 

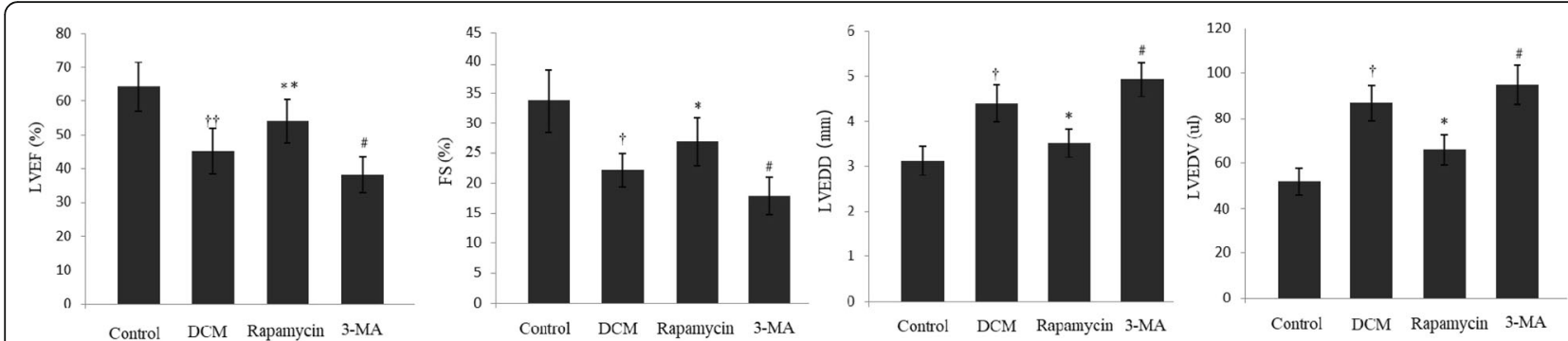

Fig. 5 Modulating autophagy and cardiac function. (A) and (B) Cardiac function differed in the four groups, and the LVEF and FS significantly improved in the rapamycin group compared with the DCM group. (C) and (D) The LVEDD and LVEDV were significantly decreased in the 3-MA group compared with the DCM group. ${ }^{\dagger+} P<0.01$ and ${ }^{\dagger} P<0.05$ vs Control, ${ }^{* *} P<0.01,{ }^{*} P<0.05$, and ${ }^{\#} P<0.05$ vs DCM. Each group, $n=8$

Echocardiography showed that the LVEF and FS significantly decreased in the DCM group, which is consisted with the pathological development of DCM. With the administration of 3-MA, cardiac function failed to improve compared with the DCM group. In the rapamycin group, cardiac function significantly improved compared with the DCM group, although the LVEF and FS were still lower compared with the control group. Meanwhile, the LVEDD and LVEDV statistically reduced by down-regulation of mTOR4EBP1 pathway to activate autophagy.

The molecular mechanisms of autophagy regulation remain unclear, an in-depth study of mTOR4EBP1 pathway might thus contribute to provide an exciting therapeutic strategy for DCM [24-26]. Our data indicated that rapamycin down-regulated the mTOR-4EBP1 signaling pathway in DCM mice. To confirm the effect of autophagy inhibition, 3-MA was employed into the present study, which decreased the formation of autophagosomes and activated the pathway as indicated by increase of $p$ mTOR and p-4EBP1 expression. Data in all demonstrated that directly targeting on the mTOR-4EBP1 pathway was a possible mechanism in the regulation of autophagy in DCM.

Our study was carefully designed and conducted in animal experimental center of Fudan University. Both up- or down-regulation of autophagy were studied by administration of rapamycin or 3-MA in parallel. Some limitations of this study should be acknowledged. We preliminary explored the mechanisms for improving cardiac function induced partially by regulating the mTOR-4EBP1 pathway, so other aspects of critical molecular mechanisms should be focused in the future research and is needed to develop better pharmacological interventions. Through further research, a more complete picture of the molecular mechanism and regulation of autophagy will strengthen our understanding of the pathological process.

\section{Conclusions}

The present study indicated that autophagy activation was involved in the pathological progress of experimental DCM. As a possible molecular mechanism, upregulating autophagy contributed to improve cardiac function in part through mTOR-4EBP1 pathway, which could be a promising therapeutic strategy for DCM.

\section{Abbreviations}

3-MA: 3-methyadenine; CVF: Collagen volume fraction;; DCM: Dilated cardiomyopathy; FS: Fractional shortening; LVEDD: Left ventricular enddiastolic dimension; LVEDV: Left ventricular end-diastolic volume; LVEF: Left ventricular ejection fraction; LVESD: Left ventricular end-systolic dimension; SD: Standard deviation; TEM: Transmission electron microscopy

\section{Acknowledgements}

We gratefully appreciate Kun Xie for her editorial suggestions for our study. The principal investigator had full access to all of the data in the present study and takes responsibility for the statistical analysis.

\section{Authors' contributions}

BJ and BWW provided the hypothesis, and handled funding and supervision. HMS, JZ and GSQZ collected and analyzed the data. BWW drafted and revised the manuscript. All authors approved the final version to be published

\section{Funding}

This study was supported in part by grants from the National Natural Science Foundation in China (No. 81470496 and No. 81800330). The funding body played no role in the design of the study, data collection and analysis, interpretation of data or in writing the manuscript.

\section{Availability of data and materials}

All relevant data is presented in the manuscript and supporting materials. The datasets used and analysed during the current study are available from the corresponding author on reasonable request.

\section{Ethics approval}

All animal studies were approved by the Animal Care and Utilization Committee of Fudan University (201802021S).

\section{Consent for publication}

Not applicable.

\section{Competing interests}

The authors declare that they have no competing interests.

\section{Author details}

${ }^{1}$ Department of Cardiology, Huashan Hospital, Fudan University, 12 Middle Urumqi Road, Shanghai 200040, China. ${ }^{2}$ Department of Medicine, Medical College of Tibet University, Lasa, Tibet, China. 
Received: 24 October 2019 Accepted: 29 January 2020

Published online: 04 February 2020

\section{References}

1. Takano H, Hasegawa H, Nagai T, Komuro I. Implication of cardiac remodeling in heart failure: mechanisms and therapeutic strategies. Intern Med. 2003:42:465-9.

2. Balmforth C, Simpson J, Shen L, Jhund PS, Lefkowitz M, Rizkala AR, et al. Outcomes and effect of treatment according to etiology in HFrEF: an analysis of PARADIGM-HF. JACC Heart Fail. 2019;7:457-65.

3. Weintraub RG, Semsarian C, Macdonald P. Dilated cardiomyopathy. Lancet. 2017:390:400-14.

4. Deshmukh V, Wang J, Martin JF. Leading progress in heart regeneration and repair. Curr Opin Cell Biol. 2019;61:79-85.

5. Frangogiannis NG. The extracellular matrix in ischemic and nonischemic heart failure. Circ Res. 2019;125:117-46.

6. Mialet-Perez J, Vindis C. Autophagy in health and disease: focus on the cardiovascular system. Essays Biochem. 2017;61:721-32.

7. Yu SY, Liu L, Li P, Li J. Rapamycin inhibits the mTOR/p70S6K pathway and attenuates cardiac fibrosis in adriamycin-induced dilated cardiomyopathy. Thorac Cardiovasc Surg. 2013;61:223-8.

8. Saito T, Asai K, Sato S, Hayashi M, Adachi A, Sasaki Y, et al. Autophagic vacuoles in cardiomyocytes of dilated cardiomyopathy with initially decompensated heart failure predict improved prognosis. Autophagy. 2016; 12:579-87.

9. Xu K, Liu XF, Ke ZQ, Yao Q, Guo S, Liu C. Resveratrol modulates apoptosis and autophagy induced by high glucose and palmitate in cardiac cells. Cell Physiol Biochem. 2018;46:2031-40.

10. Zhang X, Wang Q, Wang X, Chen X, Shao M, Zhang Q, et al. Tanshinone IIA protects against heart failure post-myocardial infarction via AMPKs/mTORdependent autophagy pathway. Biomed Pharmacother. 2019;112:108599.

11. Maeda H, Nagai H, Takemura G, Shintani-Ishida K, Komatsu M, Ogura S, et al. Intermittent-hypoxia induced autophagy attenuates contractile dysfunction and myocardial injury in rat heart. Biochim Biophys Acta. 2013;1832:1159-66.

12. Jin B, Zhu J, Shi HM, Wen ZC, Wu BW. YAP activation promotes the transdifferentiation of cardiac fibroblasts to myofibroblasts in matrix remodeling of dilated cardiomyopathy. Braz J Med Biol Res. 2019;52:e7914.

13. Basso C, Czarnowska E, Della Barbera M, Bauce B, Beffagna G, Wlodarska EK, et al. Ultrastructural evidence of intercalated disc remodelling in arrhythmogenic right ventricular cardiomyopathy: an electron microscopy investigation on endomyocardial biopsies. Eur Heart J. 2006;27:1847-54.

14. Wu B, Li J, Ni H, Zhuang X, Qi Z, Chen Q, et al. TLR4 activation promotes the progression of experimental autoimmune myocarditis to dilated cardiomyopathy by inducing mitochondrial dynamic imbalance. Oxidative Med Cell Longev. 2018;2018:3181278.

15. Ravanan P, Srikumar IF, Talwar P. Autophagy: the spotlight for cellular stress responses. Life Sci. 2017;188:53-67.

16. Yu L, McPhee CK, Zheng L, Mardones GA, Rong Y, Peng J, et al. Termination of autophagy and reformation of lysosomes regulated by mTOR. Nature. 2010;465:942-6.

17. Dunlop EA, Tee AR. mTOR and autophagy: a dynamic relationship governed by nutrients and energy. Semin Cell Dev Biol. 2014;36:121-9.

18. Zhai X, Qin Y, Chen Y, Lin L, Wang T, Zhong X, et al. Coxsackievirus B3 induces the formation of autophagosomes in cardiac fibroblasts both in vitro and in vivo. Exp Cell Res. 2016;349:255-63.

19. Shibutani S, Okazaki H, Iwata H. Dynamin-dependent amino acid endocytosis activates mechanistic target of rapamycin complex 1 (mTORC1). J Biol Chem. 2017;292:18052-61.

20. Galluzzi L, Bravo-San Pedro JM, Levine B, Green DR, Kroemer G. Pharmacological modulation of autophagy: therapeutic potential and persisting obstacles. Nat Rev Drug Discov. 2017;16:487-511.

21. Rabanal-Ruiz Y, Otten EG, Korolchuk VI. mTORC1 as the main gateway to autophagy. Essays Biochem. 2017;61:565-84

22. Xie K, Jin B, Li Y, Luo X, Zhu J, Ma D, et al. Modulating autophagy improves cardiac function in a rat model of early-stage dilated cardiomyopathy. Cardiology. 2013;125:60-8.

23. Chen Q, Zhou Y, Richards AM, Wang P. Up-regulation of miRNA-221 inhibits hypoxia/reoxygenation-induced autophagy through the DDIT4/ mTORC1 and Tp53inp1/p62 pathways. Biochem Biophys Res Commun. 2016;474:168-74.
24. Bartlett JJ, Trivedi PC, Yeung P, Kienesberger PC, Pulinilkunnil T. Doxorubicin impairs cardiomyocyte viability by suppressing transcription factor EB expression and disrupting autophagy. Biochem J. 2016;473:3769-89.

25. Koleini N, Kardami E. Autophagy and mitophagy in the context of doxorubicin-induced cardiotoxicity. Oncotarget. 2017:8:46663-80.

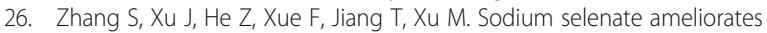
cardiac injury developed from high-fat diet in mice through regulation of autophagy activity. Sci Rep. 2019;9:18752.

\section{Publisher's Note}

Springer Nature remains neutral with regard to jurisdictional claims in published maps and institutional affiliations.
Ready to submit your research? Choose BMC and benefit from:

- fast, convenient online submission

- thorough peer review by experienced researchers in your field

- rapid publication on acceptance

- support for research data, including large and complex data types

- gold Open Access which fosters wider collaboration and increased citations

- maximum visibility for your research: over $100 \mathrm{M}$ website views per year

At BMC, research is always in progress.

Learn more biomedcentral.com/submissions 